\title{
COMMISSION 9: INSTRUMENTS AND TECHNIQUES (INSTRUMENTS ET TECHNIQUES)
}

\author{
PRESIDENT: J. Davis
}

VICE-PRESIDENT: J.C. Bhattacharyya

ORGANISING COMMITTEE: E.E. Becklin, M. Cullum, J-L. Heudier, C.M. Humphries, G. Lelievre, E.H. Richardson, L.I. Snezhko, W.J. Tango, R.G. Tull.

\section{INTRODUCTION}

The period covered by this report has seen significant progress in the development of the new generation of telescopes with apertures in the $8 \mathrm{~m}$ plus range. The period has encompassed the major construction phase of the $10 \mathrm{~m} \mathrm{Keck}$ Telescope, witnessed the commissioning of the European Southern Observatory's (ESO) New Technology Telescope and the approval of funding for the ESO Very Large Telescope (VLT). Significant progress has been achieved in developing the necessary technology for manufacturing and figuring large mirrors. There have been major expansions of activity in the areas of active control of telescope optics and adaptive optics, and in high angular resolution interferometry with several new groups entering both fields. The use of optical fibers, particularly in the area of multiple-object spectroscopy, has continued to grow. Several telescopes can now be operated remotely and the control systems of new telescopes are being designed to facilitate remote operation.

The Commission's interests have been well covered by a wide range of international meetings and workshops and the following list of proceedings of meetings gives a measure of the activity in the areas of instruments and techniques. Included in the list is the meeting co-sponsored by Commission 9 (IAU Colloquium No. 123, sponsored jointly with Commissions 44 and 48 ).

[1] "Instrumentation for Ground-Based Optical Astronomy, Present and Future; The Ninth Santa Cruz Summer Workshop", Lick Observatory (U.S.A.), 13-24 July 1987. (L.B. Robinson, Ed., Springer-Verlag).

[2] "Observatories in Earth Orbit and Beyond", Greenbelt (Maryland, U.S.A.), 24-27 April 1990. (IAU Colloquium No. 123).

[3] "Very Large Telescopes and their Instrumentation", Garching (FRG), 21-24 March 1988. (M.-H. Ulrich, Ed., ESO Conference and Workshop Proceedings No. 30)

[4] "New Technologies for Astronomy", Paris (France), 24-28 April 1989. (J.-P. Swings, Ed., Proc. SPIE 1130).

[5] "Instrumentation in Astronomy VI", Tucson (Arizona, U.S.A.), 11-17 February 1990. (D.L. Crawford, Ed., Proc. SPIE 1235).

[6] "Advanced Technology Optical Telescopes IV", Tucson (Arizona, U.S.A.), 11-17 February 1990. (L.D. Barr, Ed., Proc. SPIE 1236).

In addition to the above list, a number of meetings specialising in the astronomical applications of active and adaptive optics, optical fibers and detectors, and in high angular resolution interferometry and imaging were held. References to the proceedings of these meetings are given in the appropriate sections of this report. In total, seventeen international meetings or workshops are referenced. The proceedings of these meetings have been given an identification number since they are cross-referenced from sections other than the one in which they are listed. One result of the large number of meetings is that the main developments in astronomical instrumentation and techniques are covered extremely well by the published proceedings. For this reason only a few key references outside the numbered sequence of proceedings is given in this report.

A Commission 9 Newsletter has been started with the first issue being distributed in February and the second in August 1990 to all members of the Commission. The purpose is to keep members informed of meetings and preparations for the next General Assembly of the IAU as well as to circulate items of general interest. 


\section{TELESCOPES}

\subsection{Telescopes commissioned, under construction or planned}

A number of telescopes with apertures up to and including $4.2 \mathrm{~m}$ diameter have been commissioned and significant advances have been made in the development of a new generation of telescopes with apertures in excess of $6 \mathrm{~m}$ diameter. The main features which distinguish most new telescopes from earlier generations of telescopes are altazimuth mountings and lightweight mirrors. In many cases the designs are influenced by the application of optical fibers to multiple-object spectroscopy and the resultant desire for wide fields. There is also an increasing trend towards remotely controlled operation and most new telescopes are being built with this capability in mind.

In this report a brief outline of new telescopes, starting with those commissioned or under construction, followed by those in the design study or planning phase, will be given. More detailed information on many of these telescopes can be found in the proceedings labelled [6].

The Vatican Advanced Technology Telescope (VATT) is nearing completion and is expected to be installed on Mount Graham, Arizona, in 1990. Although it has only a modest aperture of $1.8 \mathrm{~m}$, this telescope is of particular interest because of its $\mathrm{f} / 1$ primary and altazimuth mounting which lead to a very compact design. The mirror is one of the first to be spin-cast in the Steward Observatory Mirror Laboratory at the University of Arizona and it is being figured using a new technique under development in the Mirror Laboratory known as stressed-lap polishing.

The $2.4 \mathrm{~m}$ Hiltner telescope at the Michigan-Dartmouth-MIT Observatory on Kitt Peak, Arizona, was dedicated in 1989 and the $2.56 \mathrm{~m}$ Nordic Optical Telescope (NOT) at the Roque de los Muchachos Observatory in the Canary Islands was inaugurated in September 1990. The result of collaboration between Denmark, Finland, Norway and Sweden, NOT is an $\mathrm{f} / 2$ Cassegrain telescope on an altazimuth mounting. It has a thin primary mirror supported by actuators to maintain its figure and 0.5 arcsecond resolution is claimed to be routinely obtained.

Another telescope nearing completion is the Astrophysical Research Consortium's (ARC) $3.5 \mathrm{~m}$ telescope at Apache Point, New Mexico. The ARC is a consortium comprising the Universities of Washington and Chicago, Princeton University, and New Mexico and Washington State Universities. The ARC telescope is the lightest telescope in terms of the ratio of weight to collecting area yet built. It owes this claim in large part to its fast (f/1.75), honeycomb, spin-cast mirror from the Steward Observatory Mirror Laboratory. The result is a compact altazimuth mounted telescope. An important feature of this telescope is its ability to carry as many as 9 instruments mounted around the primary, which can be selected by means of a rotatable tertiary mirror, as well as instruments at the two Nasmyth foci. Another important feature is the telescope control system which is discussed in Section 3.4.

The 4.2 $\mathrm{m}$ William Herschel Telescope (WHT) at the Roque de los Muchachos Observatory achieved first light on 1 June 1987. The telescope, on an altazimuth mount, has an $\mathrm{f} / 11$ Cassegrain and two $\mathrm{f} / 11$ Nasmyth foci. Although its mirror is thinner than most large mirrors to date, with a ratio of diameter to thickness of eight, it is also possibly the last large telescope with a thick mirror. Grubb Parsons figured the mirror to concentrate $85 \%$ of the light from a point source into a circle of diameter 0.3 arcseconds, which is better than any non-actively controlled mirror of comparable size. In addition to a range of instrumentation, which includes an echelle spectrograph and an imaging Fabry-Perot interferometer, the WHT features a Ground-based High-Resolution Imaging Laboratory (GHRIL) at one of its Nasmyth foci. This laboratory is used for interferometry and image-sharpening experiments. One experiment involving the combination of selected short exposures has produced pictures with resolutions down to 0.2 arcseconds.

The European Southem Observatory's (ESO) $3.58 \mathrm{~m}$ New Technology Telescope (NTT), located at La Silla in Chile, represents a significant advance in telescope design and performance. It achieved first light on $22 \mathrm{March} 1989$ with CCD images being transmitted in near real-time via satellite to ESO headquarters in Garching, West Germany. The NTT is a testbed for innovations for the ESO Very large Telescope (VLT) but it is a remarkable instrument in its own right. It is the first large telescope to use active control of its optics and it has been built at approximately one third of the cost of ESO's conventional $3.6 \mathrm{~m}$ telescope. The active-optics control system is outlined in Section 3.3. The NTT has Ritchey-Chrètien optics and two Nasmyth focal positions.

The construction of the $10 \mathrm{~m}$ Keck telescope, which is a joint project of the University of California and the California Institute of Technology, has continued steadily. The basic optical design is a Ritchey-Chrétien system with the primary mirror composed of 36 hexagonal segments. The mirror segments are $1.8 \mathrm{~m}$ across but only $75 \mathrm{~mm}$ thick. They are being produced to six basic surface shapes using a stressed-mirror polishing technique and will be individually warped by mechanical means in the telescope to correct any errors in their figures. The individual segments will be 
aligned relative to one another by a system of actuators responding under computer control to sensors which detect edge shifts between adjacent segments. The expectation is that stellar images of 0.25 arcsecond FWHM and with $80 \%$ of the energy within a circle of diameter about 0.4 arcseconds will be achieved. The telescope is nearing completion with the dome and telescope structure completed by the end of 1989 . The first mirror segments are being positioned on the structure and first light for $25 \%$ of the collecting surface is expected in August 1990. The target for full operation is late 1991.

The decision to build the European Southern Observatory's (ESO) Very Large Telescope (VLT) was made on 8 December 1987. The telescope, which will have four separate $8 \mathrm{~m}$ altazimuth mounted telescopes, will have the collecting area equivalent to a $16 \mathrm{~m}$ telescope. The telescopes will be usable as separate telescopes or in combined mode. Interferometric operation, at least in the infrared, is planned. The configuration of the four telescopes is site dependent with a decision on the choice of site expected by the end of 1990 . Site testing is continuing in Chile with the choice lying between the established La Silla site and two potential observatory sites at Cerro Viscachos and Cerro Paranal. The completion of the first $8 \mathrm{~m}$ telescope is scheduled for 1994 and the entire VLT for 1998.

The University of Texas and Pennsylvania State University are collaborating in the construction of the Spectroscopic Survey Telescope (SST), a special-purpose telescope in which the primary mirror consists of eighty five spherically-figured $1 \mathrm{~m}$ diameter circular segments arranged in a $10 \mathrm{~m}$ diameter frame. The maximum light-gathering power is equivalent to a single $8.5 \mathrm{~m}$ diameter mirror. The primary focal length is $13 \mathrm{~m}$. The telescope is to be located on Mt. Locke in the Davis Mountains of westem Texas. The telescope superstructure holds the mirror array at a fixed tilt of $30^{\circ}$ from the zenith and is rotatable in azimuth in $2^{\circ}$ increments. The field of view in the focal surface is $\pm 6^{\circ}$, allowing the telescope to access declinations from $-5^{\circ}$ to $+67^{\circ}$. Target objects are tracked across the field by an acquisition module consisting of a two-mirror Gregorian focal-plane corrector and optical fiber cables linked to a fixed spectrograph. The average allowable observing time per object will be approximately 60 minutes. Figuring of mirror segments has commenced and the telescope is scheduled for completion in 1994.

A number of telescope projects are dependent on the Steward Observatory Mirror Laboratory (SOML) successfully spin-casting lightweight mirror blanks of increasing diameter. These include the ARC $3.5 \mathrm{~m}$ telescope already discussed, the WIN $3.5 \mathrm{~m}$ telescope project (a collaboration between the National Optical Astronomy Observatory (NOAO), the University of Wisconsin, and Indiana University), upgrading of the Multi-Mirror Telescope (MMT), the Columbus and Magellan telescope projects, and NOAO plans for two $8 \mathrm{~m}$ telescopes in partnership with organisations yet to be determined.

The WIN $3.5 \mathrm{~m}$ Telescope Project is at the design stage although the primary mirror, the second $3.5 \mathrm{~m}$ spin-cast blank from SOML, is being polished to a sphere in preparation for thermal and support system testing. In the design, emphasis is being placed on the provision of a wide field of view for multiple-object spectroscopy. The telescope is to be installed on Kitt Peak, Arizona.

Plans to replace the six $1.8 \mathrm{~m}$ diameter mirrors of the Multi-Mirror Telescope (MMT) with a single $6.5 \mathrm{~m}, \mathrm{f} / 1.25$ mirror await the spin-casting of the mirror blank at SOML, scheduled for February 1991. When installed, the effective collecting area of the telescope will be increased by a factor of two from the equivalent of a $4.5 \mathrm{~m}$ diameter aperture.

The Columbus telescope project is a collaboration between the Osservatorio Astrofisico di Arcetri of Florence, the Ohio State University, and the University of Arizona. The design features two $8 \mathrm{~m}$ diameter $\mathrm{f} / 1.2$ telescopes mounted side-by-side to give an effective collecting area equivalent to $11.3 \mathrm{~m}$ diameter and a maximum baseline of $22 \mathrm{~m}$ for interferometry. The design of the binocular telescope has been completed and construction is to commence. The telescope is to be located on Mt. Graham, Arizona. The plan is to complete a "one-eyed" telescope by 1994 and the full binocular system by 1996. The project is dependent on the successful spin-casting and figuring of $8 \mathrm{~m}$ blanks by SOML.

The Magellan Project is a joint enterprise of the Camegie Institution of Washington, the Johns Hopkins University, and the University of Arizona to build an $8 \mathrm{~m}$ telescope at Camegie's Las Campanas Observatory in Chile. The University of Arizona will contribute the $8 \mathrm{~m}$ diameter, $\mathrm{f} / 1.2$ mirror, which will be spin-cast and figured by SOML. Commencement of final design and construction requires feasibility demonstrations of all key components and a complete funding plan. This includes the successful polishing of the VATT $1.8 \mathrm{~m}$ mirror to $\mathrm{f} / 1$ and the successful casting of the $6.5 \mathrm{~m}$ mirror for the MMT upgrade by SOML.

NOAO is seeking funding for building two $8 \mathrm{~m}$ altazimuth mounted telescopes, one to be located on Mauna Kea, Hawaii and the other at Cerro Pachon, Chile. The possibility of international partners in the project is being explored. The telescopes are planned to provide high quality imaging $(0.25$ arcseconds), to have provision for adaptive optics, and 
the capability for future use in interferometric arrays. They will cover a field of view up to 45 arcminutes. There are plans for a full range of imaging and spectrographic instrumentation covering the optical and infrared. Both telescopes would use spin-cast, lightweight, honeycomb monolithic mirrors from SOML.

Japan is planning to build an optical/infrared $7.5 \mathrm{~m}$ altazimuth mounted telescope on Mauna Kea, Hawaii. To be known as the Japanese National Large Telescope the project is at the design study stage. Instruments planned for the telescope include a high resolution echelle spectrograph at one of the Nasmyth foci and a multiple-object spectrograph for the Cassegrain focus.

Reviews of astronomy in West Germany and Britain have both recommended that the respective countries should build large reflecting telescopes. The German report recommended that the German Large Telescope (DGT) should have an aperture of $12 \mathrm{~m}$ and the British plan to build an $8 \mathrm{~m}$ optical-infrared telescope if a suitable international partner can be found. Design studies for an altazimuth mounted telescope have been commenced by the British.

For telescopes of modest aperture a novel approach, called a Multi-Telescope Telescope (MTT), has been proposed (Bagnuolo et al. 1990). The prototype of this approach, which takes advantage of optical fibers, is being constructed by the Centre for High Angular Resolution Astronomy of Georgia State University. In this design a number of small telescopes of aperture diameter $33 \mathrm{~cm}$, using commercially available paraboloids for their primaries, are mounted in parallel in a single altazimuth mount. A fiber is mounted at the focus of each of the telescopes $(9$ in the prototype) to feed light to an off-telescope spectrograph. The cost is estimated to be a small fraction of that of a conventional telescope of comparable collecting area.

The first routine operation of an automatic photoelectric telescope (APT) commenced in 1983 and since then a number of small telescopes have been installed to provide automatic photometry and photometric monitoring of selected objects. In February 1988 a new 0.75 m APT was unveiled on Mount Hopkins where it joined three smaller reflectors that operate unattended on every clear night. Automated telescopes are ideal for repetitious observations such as filter photometry and CCD imaging and additional telescopes, typically in the $1 \mathrm{~m}$ class, are being built or planned. There are plans to link such telescopes into a Global Network to coordinate observations and to obtain long time coverage.

\subsection{Solar telescopes (Prepared by W.C. Livingston)}

A new general research instrument that aims at high spatial resolution on the solar disk is the Kiepenheuer Vacuum Tower Telescope on Tenerife in the Canary Islands. Operations began in 1989 and the $0.7 \mathrm{~m}$ aperture $76 \mathrm{~m}$ focal length optics are providing excellent images (a matter of concern these days). Another instrument is the French polarimetric Télescope Héliographique pour l'Étude du Magnétisme et des Instabilités Solaires (THEMIS). This is under construction at nearby La Palma although complete funding is yet to be realized. Likewise the polarimetric Large Earth-based Solar Telescope (LEST), also to be sited at La Palma, is at the time of writing not financially secure although preliminary designs are finished. At the U.S. National Solar Observatory (NSO) an upgrade of the $1.6 \mathrm{~m}$ McMath Telescope to $4 \mathrm{~m}$ is proposed. This larger aperture would permit diffraction limited solar observations in the $\operatorname{IR}$ at $12 \mu \mathrm{m}$. Feasiblity studies for a super-polished all-reflecting coronagraph is another NSO project. An ambitious Solar Cycle Telescope is under consideration at Mitaka.

Small telescopes for 24-hour synoptic observations of solar oscillations are being developed. One global network consists of stations at Kunming, Kislovodsk, and Tucson. Although the telescopes have only $7 \mathrm{~cm}$ apertures, their instrumentation is state-of-the-art. The Global Oscillation Network Group (GONG) is a six station network for oscillation studies. A GONG prototype instrument is presently producing engineering data in Tucson.

The proposal for a world-wide system of standardized solar synoptic telescopes, with accompanying provision for data exchange, received much discussion and interest in Commissions 10 and 12 at the Baltimore IAU General Assembly. Scientific returns from such a network would provide invaluable information on the sun, but a realistic plan for funding is yet to evolve.

\subsection{Telescope mirror manufacture}

The challenge of providing accurate reflecting surfaces, which remain true with telescope orientation, for telescopes with apertures up to $8 \mathrm{~m}$ in diameter is providing competition between two main schools of thought. One is tackling the problem by using thin, solid mirrors whose shape is maintained by closed-loop actuator systems. The development of this technique is being led by ESO who have demonstrated its successful operation in their $3.58 \mathrm{~m}$ NTT. ESO is planning to employ the technique in the $8 \mathrm{~m}$ telescopes of the VLT. The alternative approach, promoted by J.R.P. 
Angel and the Steward Observatory Mirror Laboratory (SOML), is to spin-cast mirrors with an integral honeycomb support structure which, it is claimed, will hold the surface shape without the need for active correction. This latter approach is at an earlier stage of development with three $3.5 \mathrm{~m}$ diameter mirror castings being optically worked. Both approaches use lightweight mirrors giving the advantages of lighter support structures and reduced costs. The proponents of both techniques agree that for reflecting surfaces larger than $8 \mathrm{~m}$ in diameter it will be necessary to consider a segmented primary mirror. Pioneering work in this area is being performed for the $10 \mathrm{~m}$ Keck telescope.

SOML has a program to spin-cast mirrors in a series of size steps before it attempts its ultimate aim of producing $8 \mathrm{~m}$ diameter mirror blanks. The mirrors, with an integral honeycomb support structure, are cast from borosilicate (Pyrex like) glass and the first success was a $1.2 \mathrm{~m}$ diameter blank for the Smithsonian Astrophysical Observatory in November 1987. Since then a number of castings have been made including a $1.8 \mathrm{~m}$ diameter $\mathrm{f} / 1$ blank for the Vatican Advanced Technology Telescope and three $3.5 \mathrm{~m}$ blanks. The latter include the $\mathrm{f} / 1.75$ disks for the ARC and WIN telescope projects.

The SOML furnace is being enlarged to its full diameter of $10 \mathrm{~m}$ in readiness for spin-casting $6.5 \mathrm{~m}$ and $8 \mathrm{~m}$ mirror blanks and the laboratory is being modified and expanded to accomodate two $8 \mathrm{~m}$ capacity polishing machines and a rigid, vibration-isolated test tower. Future plans include the $6.5 \mathrm{~m} \mathrm{f} / 1.25$ blank for the MMT upgrade and, if this is successful, $8 \mathrm{~m}$ blanks for a number of telescopes including the Columbus, Magellan and NOAO projects.

Schott Glaswerke of West Germany have also developed a technique for spin-casting solid Zerodur blanks and have made mirror blanks up to $4 \mathrm{~m}$ in diameter. They have been given the contract to spin-cast four Zerodur blanks for the VLT. The blanks will be $8.2 \mathrm{~m}$ in diameter but only $175 \mathrm{~mm}$ thick.

An alternative approach to producing lightweight mirrors is being pursued at the University of Arizona's Optical Sciences Center. The mirror is all metal with a thick disc of aluminium foam sanndwiched between two thin aluminium faceplates. The structure adapts to changes in ambient air temperature relatively rapidly. To date only small mirrors of a few tens of centimeters in diameter have been made.

In addition to the innovative work in producing large blanks considerable effort has gone into developing techniques for polishing aspheric mirror segments and the fast conic sections made feasible by the spin-casting technique. In the former case a technique known as stressed-mirror polishing has been developed for the Keck telescope's mirror segments. The thin mirror segments are stressed to a predetermined shape during polishing. They are polished to a spherical surface such that when the stresses are removed the surface has the required off-axis aspheric shape. Because the blanks can only be stressed successfully as circular disks they are cut to their final hexagonal shape after polishing. This releases stresses and results in some deformation which is being corrected in the Keck telescope by warping the mirror segments in the telescope to the desired shape by mechanical means. The Keck segments are $1.83 \mathrm{~m}$ wide hexagons cut from $2.13 \mathrm{~m}$ diameter circular disks $75 \mathrm{~mm}$ thick. A different approach is being developed by SOML for the very fast primary mirrors it is producing with the spin-casting method. Known as stressed-lap polishing it uses a lap whose shape is continually adjusted to match the desired aspheric surface as it moves across it. The technique is being perfected during the polishing of the Vatican Advanced Technology Telescope's $1.8 \mathrm{~m} \mathrm{f} / 1$ primary mirror. The $75 \mathrm{~cm}$ lap has 12 computer controlled actuators and its shape is adjusted at rates up to 2000 times per second. It is planned to use this technique on spin-cast mirrors of $8 \mathrm{~m}$ diameter.

Further information and details of the advances in mirror production outlined above can be found in the proceedings labelled [6].

\section{INSTRUMENTATION AND TECHNIQUES}

It is not feasible to give a comprehensive review of all new instruments and developments in instrumentation and techniques. Instead a few key areas where significant progress has been made will be outlined.

\subsection{Instruments}

The common elements in the instrumentation plans for most of the new generation of large aperture telescopes outlined in Section 2.1 include high resolution echelle spectrographs, multi-object optical fiber linked spectrographs, and infrared imaging and spectrographic instruments taking advantage of the infrared array detectors now available. 
Most existing large telescopes as well as the new generation of large telescopes are being equipped with large echelle spectrographs. An example of a state-of-the-art high resolution spectrograph is the University College London Echelle Spectrograph (UCLES) installed on the $3.9 \mathrm{~m}$ Anglo-Australian Telescope (AAT). This instrument employs a large echelle grating with a train of fused silica cross-dispersing prisms to give a spectral resolution of 80,000 down to magnitude 16.5 at wavelengths from 300 to $100 \mathrm{~nm}$. It is installed at the AATs coude focus but it is planned to feed it via a single optical fiber from the Cassegrain focus. The UCL team is collaborating in the development of a version of UCLES for the Nasmyth focus of the $4.2 \mathrm{~m}$ WHT.

Multi-object, optical fiber linked spectrographs are discussed in Section 3.2 but it is noted that the development of fully automated and remotely operated fiber positioning systems with increasing numbers of fibers is having a profound influence on telescope design with much emphasis being placed on large diameter fields of view.

A new generation of infrared spectrographs has become possible with the availability of multiplexed infrared array imaging detectors eliminating the need for sequential scanning. An example is IRSHELL, an infrared cryogenic echelle spectrograph (Lacy et al. 1989). The instrument uses a liquid-helium-cooled echelle grating and $10 \times 64$ pixel Si:As impurity band detector array to obtain a 64 point spectrum with resolving power of 1000 to 25,000 at each of 10 points spaced by 0.4 to 3 arcseconds along a slit. Spectra have been obtained at wavelengths between 4 and $17 \mu \mathrm{m}$. Instruments of this general form are being designed for most of the new generation of large telescopes.

Further details and additional examples of instruments built or under development can be found in the proceedings labelled [1], [3] and [5].

\subsection{Optical fibers}

The use of optical fibers in astronomical applications continues to grow and most major observatories have instruments employing them. Astronomical observations are being revolutionised in many cases and this, in turn, is impacting on telescope design and the upgrading of existing telescopes. Fibers with improved characteristics for astronomical applications, in particular improved uv-blue transmission and reduced focal ratio degradation (FRD), have become available through small companies producing fibers for instrumentation applications. The first conference devoted to optical fibers in astronomy has been held and the proceedings published:

[7] "Fiber Optics in Astronomy", Tucson (Arizona, U.S.A.), 11-14 April 1988. (S.C. Barden, Ed., Astron. Soc. Pac. Conf. Ser. 3).

In addition, most conferences on telescopes and instrumentation now include contributions involving the use of optical fibers.

A major area of activity is the development and use of multiple-object spectrographs in which fibers are used to relay the light from several images in the focal plane of the telescope simultaneously to the spectrograph slit. Apart from the obvious advantage of increasing the observing efficiency of telescopes by up to two orders of magnitude or more, fibers give greater freedom in the location of large spectrographs in convenient and stable environments. While several instruments with fully automated and remotely operated fiber positioning systems were in operation at the time of the 1984-87 report, significant developments have since taken place. The trend has been towards improved operational efficiency in the fiber positioning systems and towards increased numbers of fibers to allow more objects to be observed simultaneously. The increase in the number of fibers has brought a demand for wider fields and the newer instruments have more fibers and cover wider fields. Examples include a second-generation version of the $3.8 \mathrm{~m}$ Anglo-Australian Telescope's automated and remotely controlled fiber positioning system (Autofib) for the $4.2 \mathrm{~m}$ William Herschel Telescope ( 300 fibers; 1 degree diameter field), the fiber-fed Norris spectrograph of the $5 \mathrm{~m}$ Hale telescope (176 fibers; 20 arcminute diameter field), a new fiber feed system for the Kitt Peak National Observatory $4 \mathrm{~m}$ Mayall telescope (100 fibers; 45 arcminute diameter field) and a system under development for the Lick $3 \mathrm{~m}$ telescope (100 fibers; 1 degree diameter field). A prime example of the trend is a major instrument under development for the Anglo-Australian Telescope. The new instrument, known as $2 \mathrm{dF}$ ( 2 degree Field), is specifically designed for wide-field, multiple-object spectroscopy. The instrument is in fact a dedicated prime focus assembly which includes a field corrector combined with dedicated fiber-fed spectrographs (400 fibers; 2 degree diameter field). Further details can be found in conference proceedings [5] and [7].

Optical fibers are being used increasingly to couple telescopes to instruments in fixed, stable locations. This is particularly attractive in the case of telescopes of modest aperture size and applications include precision radial velocity measurements and high resolution spectra of bright objects. 
The multiplex advantage of fibers in precision photometry is attracting attention but the problems associated with obtaining stable transmission efficiency requires further work. Effects such as stress-induced FRD varying with telescope attitude and transmission variations resulting from small movements of the stellar image on the fiber input face have to be overcome.

The properties of single-mode fibers have been shown to make them ideal for use in a multi-aperture spatial interferometer mounted on a large steerable structure and work on multimode fibers for long baseline interferometry shows promise.

\subsection{Active/adaptive optics}

The field of active and adaptive optics has seen major progress within the last few years with ESO scoring two very significant firsts. ESO's NTT is the first large telescope to use active control of its optics and the first diffraction-limited astronomical images using adaptive optics were obtained on the ESO $3.6 \mathrm{~m}$ telescope.

The NTT has active control on both its primary and secondary mirrors. The shape of the primary mirror and the position of the secondary mirror are controlled by closed-loop systems to optimise the optical performance of the telescope during observations. While corrections can be made with a cycle time of a few minutes, in practice it is found to be necessary only once an hour. The primary has 3 fixed supports and 75 active supports and the secondary is adjusted for centering and focus. The optical system can be maintained near the intrinsic quality in which $80 \%$ of a star's light is concentrated in a circle of 0.15 arcsecond diameter. Stellar images of FWHM equal to 0.35-0.39 arcseconds have been recorded and a double star with a separation of 0.79 arcseconds has been completely resolved.

A prototype adaptive optics system for the European Southern Observatory (ESO) Very Large Telescope (VLT) has been developed in a collaboration between ESO, the Observatoire de Meudon, ONERA and Laserdot in France. Successful tests were carried out in the laboratory in September 1989 and on the Observatoire de l'Haute Provence $1.52 \mathrm{~m}$ telescope in October-November 1989. Diffraction limited images at a wavelength of $3.5 \mu \mathrm{m}$ were obtained at the ESO $3.6 \mathrm{~m}$ telescope at La Silla in April 1990 (Rousset et al. 1990). The system was based on a Shack-Hartmann wavefront sensor and a 19 actuator deformable mirror. The system employed a modal correction algorithm and operated with a $10 \mathrm{~Hz}$ bandwidth (sampling at $100 \mathrm{~Hz}$ ).

Image stabilisation systems are being developed at a number of institutions including the University of Durham in England, ESO, and the Dominion Astrophysical Observatory (DAO) in Canada. At DAO, a high-resolution imaging camera system is being developed for the $3.6 \mathrm{~m}$ Canada-France-Hawaii Telescope (CFHT) on Mauna Keea, Hawaii. The Mark I system uses a small, flat, piezo-actuated tilting mirror to stabilise the image position. The system also includes a fast shutter for selecting intervals of good seeing and optional diaphragms to further improve the resolution. Preliminary tests indicate that images with a resolution of 0.2 arcseconds can be frequently obtained. A Mark II system is being designed in which the correcting mirror will have eight pie-shaped segments to correct individual parts of the image separately.

The National Optical Astronomy Observatories (NOAO) Advanced Development Program, which included an active/adaptive optics program, was axed in 1988 due to financial constraints.

F. Roddier has proposed a new wavefront sensing method based on local wavefront curvature measurements rather than slope measurements (Roddier 1988). This new approach may be used in adaptive optical systems, for monitoring seeing, and for testing telescope optics. A seeing monitor based on wavefront curvature sensing has been built and tested on Mauna Kea, Hawaii. Several aspects of this technique are reported in the proceedings labelled [6].

A segmented bimorph deformable mirror has been under development at NOAO using the efficient low-voltage bending properties of PZT bimorph wafers (Forbes et al. 1989). A 37-hex-faceted deformable mirror $23 \mathrm{~mm}$ in diameter has been produced which shows promise for use in adaptive systems for atmospheric wavefront distortion correction.

The proceedings of three international meetings are key references for an overview of the subject:

[8] "Diffraction-Limited Imaging with Very Large Telescopes", Cargese (France), 13-23 September, 1988. (D.M. Alloin and J.-M. Mariotti, Eds., NATO ASI Series, Series C: Mathematical and Physical Sciences 274).

[9] "Active Telescope Systems", Orlando (Florida, U.S.A.), 28-31 March 1989. (F. Roddier, Ed., Proc. SPIE 1114). 
[10] "Adaptive Optics and Optical Structures", The Hague (Netherlands), 12-14 March 1990. (R.K. Tyson, J. Schulte in Den Baumen, Ed., Proc. SPIE 1271).

In addition, a review of adaptive optics by H.W. Babcock, who is regarded as the founder of the field, has been published (Babcock 1990). The review, although brief, covers the origins of the topic through to the latest developments and lists the key publications in the field.

\subsection{Telescope operation}

The increasing trend towards the remote control of telescopes and the fact that most new telescopes are being built with this capability in mind has already been mentioned. Examples of this trend include the $4.2 \mathrm{~m}$ WHT, ESO's $3.58 \mathrm{~m}$ NTT, and the $3.5 \mathrm{~m}$ ARC telescope.

The WHT has a computer control system which has been designed with remote operation in mind. Until remote operation is implemented it permits observers to operate the telescope and run their programs without the assistance of a telescope operator.

ESO established a satellite link between their observatory at La Silla, Chile and headquarters in Garching, West Germany in 1987. Initially used to allow remote operation of $2.2 \mathrm{~m}$ and $1.4 \mathrm{~m}$ telescopes it is being extended to provide the remote observing option for all of ESO's telescopes. The link was used during first light observations with the NTT. An observer in Garching took control of the NTT's computer and CCD images from the telescope were studied in near to real time in Germany.

The operating plans for the ARC $3.5 \mathrm{~m}$ telescope are a clear indication of the trend to remote operation and improved efficiency of telescope operation. The telescope control system, including instrument selection, can be operated by the observer from a personal computer at their home institution. The intention is to have complete flexibility in the observing programs so that they can be matched to the observing conditions. For example, it will be possible to implement programs requiring excellent seeing immediately such conditions prevail.

In order to obtain time series photometry of rapid variable stars a world-wide network of telescopes has been organised (Nather et al. 1990). The network is designed to minimise or eliminate gaps in the brightness record caused by the rotation of the Earth. The network is coordinated from a single control center and as such is equivalent to a single multiple-mirror telescope. Designated target stars are measured so long as they are in darkness, with data returned to the control center by electronic mail for near real-time analysis, thus permitting the coordinating astronomer to decide when a particular string of data should be terminated or continued. Unbroken data strings exceeding 24 hours in length have been obtained. During March 1989 such data strings were obtained using 9 telescopes at Mauna Kea (Hawaii), McDonald Observatory (Texas), Cerro Tololo (Chile), Itajuba (Brazil), La Palma (Canary Islands), Haute Provence (France), Sutherland (South Africa), Kavalur (India) and Siding Spring (Australia). The program is supported by grants from the National Science Foundation and the National Geographic Society.

\section{WORKING GROUPS}

Commission 9 has three Working Groups: Astronomical Photography, Detectors, and High Angular Resolution Interferometry. The Working Group on Astronomical Photography has a meeting of the Working Group scheduled for 29-30 October 1990 at the ESO Headquarters in Garching. Reports from the other two Working Groups follow.

\subsection{Detectors (Report by G. Lelievre, Chairman of the Working Group)}

Several international meetings with particular emphasis on detectors and their astronomical applications have been held. Published proceedings include:

[11] "Infrared Astronomy with Arrays", Hilo (Hawaii, U.S.A.), 24-26 March 1987. (C.G. Wynn-Williams, E.E. Becklin, Eds., University of Hawaii).

[12] "The Ninth Symposium on Photo-Electronic Image Devices", 7-11 September 1987. (B.L. Morgan, Ed., Advances in Electronics and Electron Physics, (in press)). 
[13] "CCDs in Astronomy", Tucson (Arizona, U.S.A.), 6-8 September 1989. (G.H. Jacoby, Ed., Astron. Soc. Pac. Conf. Ser. 8).

[14] "Charge-Coupled Devices and Solid-State Optical Devices", 12-14 February, 1990. (M. Blouke, Ed., Proc. SPIE 1242).

[15] "CCDs in Astronomy II - New Methods and Applications of CCD Technology", Charleston (South Carolina, U.S.A.), 15-17 March 1990. (A.G. Davis Philip, D.S. Hayes, S.J. Adelman, Eds., L. Davis Press, Schenectady, New York).

4.11 Infrared Arrays. The use of 2-dimensional arrays is now wide-spread amongst most of the large telescopes. Various detectors are set-up for general use, with good photometric quality and an adequate number of pixels (128 $\times$ 128). This is a significant advance compared with five years ago, when it was quite a challenge for a specialist to acquire infrared images of bright objects with small detectors (32 $\times 32$ pixels). At the Hilo meeting in 1987 [11] and at the ESO conference in 1988 [3] early observational results were reported along with instrumental problems. Subsequent progress has been spectacular and was well illustrated at the meeting in Tucson [13]. Technical topics were no longer the subject of the conference; on the contrary, the richness of observations and data presented (more than 100 contributions) demonstrated the degree of maturity reached by the field. The proceedings of this meeting may be considered to mark the birth of a new domain of astrophysical observations.

4.12 Charge Coupled Devices (CCDs). References to complete compilations on the state of CCD technology and their critical application to astronomy were given in the 1984-87 Commission 9 report. On-going research and development work aimed at better CCD sensors was presented and discussed at the ninth Santa Cruz Summer Workshop [1]. Technical problems were covered including the difficulties in producing large format sensors and coatings to improve the quantum efficiency at wavelengths shorter than $0.4 \mu \mathrm{m}$. The difficulty in obtaining scientific grade devices was discussed but the astronomical need for large area sensors with low read-out noise remained a promise yet to be fulfilled.

At the ESO conference a year later [3] the situation had not improved significantly. With the supply of large format chips uncertain, an alternative solution, involving the building of large mosaics using smaller and easier to produce "buttable" CCDs from Thomson-CSF, France, was proposed.

In the last eighteen months, significant progress has been achieved towards meeting the needs of astronomy. As a result of strong competition between industrial companies, large format CCDs have become available. Several observatories have acquired these new CCDs for testing and use on telescopes. CCDs with 1024 x 1024 pixels are now in regular use at major observatories around the world. The field is evolving very rapidly as witnessed by three recent conferences dealing with CCD astronomy and technology [13], [14], [15].

A wide range of $\mathrm{CCD}$ formats is now available ranging from $2 \times 2$ pixels (Tektronics) up to $4096 \times 4096$ pixels (Ford). Kodak offers two arrays: a 2048 × 2048 pixel full frame array and a $1024 \times 1024$ pixel interline-transfer array. These are two-phase devices with pixels $9 \mu \mathrm{m}$ square and the capability of operating at a data rate of 20 Megapixels/s. CCDs manufactured by Ford Aerospace Corp. with 2048 × 2048 pixels have been acquired and tested by several observatories including Lick, CFHT and ESO. Readout noise is in the process of being optimised (10 $\mathrm{e}^{-}$at ESO). Thinning techniques have been developed at Lick Observatory. Ford has succeeded in fabricating a $4096 \times 4096$ array with $7 \mu \mathrm{m}$ square pixels.

Tektronics have several types of CCD chips available: $512 \times 512$ pixels thick or thin, $1024 \times 1024$ pixels thick or thin with $19 \mu \mathrm{m}$ square pixels, and $2048 \times 2048$ pixels. These systems are proving to be $\mathrm{x}$-ray photon noise limited in medical imaging.

Thomson-CSF is also offering chips with $512 \times 512$ and $1024 \times 1024$ pixels (thick or thin). The readout noise of the most recent is improved and according to ESO is less than $5 \mathrm{e}^{-}$.

Reticon is preparing a $2048 \times 2048$ pixel CCD and EEV(GEC), England, have proposed a novel technique of reticle composition allowing arrays of arbitrary size in one dimension to be produced. $298 \times 1152$ pixel and $2166 \times$ 1152 arrays have been produced and very long linear arrays could be fabricated with this composition. 
Coating techniques have been mastered by several companies and observatories to improve the ultraviolet sensitivity of thick CCDs. Noise reduction remains a high priority and on-chip processing and data manipulation is another important area under consideration.

4.13 Photoelectric Detectors. Reviews of the status of photoelectronic detectors can be found in the proceedings of two meetings [1], [12].

An improved version of the intensified CCD CP 40 photon-counting camera has been completed at Paris Observatory.

New versions of the Multi-Anode Microchannel Array (MAMA) detector with larger format up to $2000 \times 2000$ pixels are being developed for space projects. A $40 \mathrm{~mm}$ dual microchannel plate image intensifier has been developed at Imperial College, London. Ranicon (Quantar) detectors are commercially available. Further information on these detectors can be found in conference proceedings [5].

Photoelectric detectors remain of high interest when time-resolution is important. In particular, with the developments anticipated in active and adaptive optics there is a crucial need for wavefront sensors of increased sensitivity in order to reach faint objects and to discriminate against multi-electron impacts. An intensified CCD system like the CP 40 is quite suitable at this time but the dynamics are low. An altemative is the electron bombarded CCD. LEP (Philips) and Paris Observatory have made encouraging tests of this approach which have been reported in the conference proceedings labelled [5].

The Precision Analogue Photon Address (PAPA) camera developed at the Harvard-Smithsonian Center for Astrophysics (CfA), Cambridge, U.S.A. is another detector particularly suited to applications where time resolution is important (Papaliolios 1985). It has found applications in speckle and long baseline interferometry where sampling times of the order of a few milliseconds are required to avoid phase smearing due to atmospheric turbulence. Three cameras have been built by Adaptive Optics Associates, Boston, and recently a further five have been built in a collaborative program involving CfA, Imperial College (London), Georgia State University, the United States Naval Observatory, and the University of Sydney. All are destined to be used in astronomical interferometry programs.

\subsection{High Angular Resolution Interferometry (Report by W.J. Tango, Chairman of the Working Group)}

Activity in the field continues to increase rapidly. Probably the most exciting development has been the move away from demonstration prototypes to large scale astronomical interferometers. In 1987, for example, there was only one instrument with a baseline longer than $50 \mathrm{~m}$. That has now been extended to $140 \mathrm{~m}$ and in addition there are several interferometers which are currently under construction which will provide baselines up to $640 \mathrm{~m}$.

Instrumentation and techniques have continued to improve and proceedings of international meetings with particular emphasis on high angular resolution interferometry and imaging include:

[16] "High Resolution Imaging by Interferometry", Garching (FRG), 15-18 March 1988. (F. Merkle, Ed., ESO Conference and Workshop Proceedings No. 29).

[17] "Amplitude and Intensity Spatial Interferometry", Tucson (Arizona, U.S.A.), 11-17 February 1990. (J.B. Breckinridge, Ed., Proc. SPIE 1237).

The proceedings of these two meetings are useful references to recent advances in the field.

To assist members of the Working Group an annual bibliography is maintained. The bibliography is restricted essentially to instrumentation and techniques and is not exhaustive, since it is compiled by the Chairman and based on responses from the members of the WG. The bibliography was not distributed in 1988, but a combined 1988-1989 bibliography will be sent to members in 1990 .

The Chairman was asked by the Working Group in Baltimore to investigate the production of an informal newsletter. A newsletter has been produced at NOAO by S.T. Ridgway in recent years and, starting in 1990, we will combine forces and produce a joint newsletter which will be sent to all members of the Working Group.

4.21 Diffraction-limited imaging with a single large aperture. The standard technique in this area is speckle interferometry, but the past few years have seen the development of non-redundant masking (NRM) techniques. In NRM inter- 
ferometry the telecope aperture is masked except for a set of subapertures approximately equal to $r_{o}$ in diameter. The resulting image is crossed by fringes produced by each pair of subapertures, and if each pair produces a unique fringe pattern the visibility moduli and closure phases can be determined. The astronomical potential of the method has recently been demonstrated (Buscher et al. 1990). It is also proving to be a useful tool for investigating the imaging characteristics of long baseline interferometers which use aperture diameters of the order of $r_{0}$.

Adaptive optics uses wavefront sensors to detect the aberration in the incoming light and deformable optical elements to compensate for the wavefront distortions. Although the concept is not new, the first diffraction-limited images using adaptive optics were obtained only in 1990 (Rousset et al. 1990). This achievement is discussed in more detail in Section 3.3.

4.22 Interferometry with two or more widely separated apertures. As noted above, there have been major developments in long baseline interferometry. Most of these have been described, at least briefly, in one of the major conference proceedings labelled [8], [16] and [17], so specific references to individual projects will not be given here.

In addition to the projects outlined below it should be noted that the interest in high angular resolution interferometry continues growing and new groups are entering the field. As an example, the Shaanxi Astronomical Observatory in China is developing plans to construct a prototype long baseline interferometer and undoubtedly there are others.

The Interférometre à 2 Télescopes (I2T) of l'Observatoire de la Côte d'Azur (OCA) (previously associated with CERGA) has been extended to a maximum baseline of $140 \mathrm{~m}$ and plans to convert it to a three aperture instrument, known as CHARON, are being implemented. A western arm is under construction. The Grand Interférometre à 2 Télescopes (GI2T), with apertures of $1.5 \mathrm{~m}$, is now working. It is also being developed into a three-element interferometer. In addition OCA operates an infrared interferometer (SOIRDETE) with a $15 \mathrm{~m}$ baseline and $1 \mathrm{~m}$ apertures.

The University of California's Infrared Spatial Interferometer (ISI) is now operational at Mt. Wilson. This is a heterodyne interferometer which has baselines ranging from $4 \mathrm{~m}$ to $34 \mathrm{~m}$ and operates at a wavelength of $10 \mu \mathrm{m}$ with an aperture diameter of $1.65 \mathrm{~m}$. A unique feature of ISI is that the input optics are mounted in trailers which can be moved between stations. The maximum baseline is therefore determined primarily by the site rather than the equipment, and ISI has been designed to work with baselines up to $1000 \mathrm{~m}$.

A smaller infrared interferometer, the InfraRed Michelson Array (IRMA), is operated by the University of Wyoming and Kitt Peak National Observatory. The baseline coverage is from $2.5 \mathrm{~m}$ to $19.5 \mathrm{~m}$ with $0.2 \mathrm{~m}$ apertures, and it is designed to operated at $2.2 \mu \mathrm{m}$.

The Sydney University Stellar Interferometer (SUSI) is virtually complete and interferometric observations are expected to commence in 1991. SUSI consists of 12 fixed siderostat stations aligned on a N-S baseline. This arrangement provides a range of baselines from $5 \mathrm{~m}$ to $640 \mathrm{~m}$. Each station is equipped with a set of input optics and the light is transmitted to the central laboratory through evacuated pipes. The maximum aperture size is $0.14 \mathrm{~m}$ and the operating wavelength is $400-800 \mathrm{~nm}$. A path compensating system consisting of two $70 \mathrm{~m}$ long precision tracks provides up to $\pm 420 \mathrm{~m}$ of (air) optical path. Although it is planned initially to operate SUSI as a two-element interferometer, provision has been made for an eastern extension so that it can be converted to a three-element instrument.

The Cambridge Optical Aperture Synthesis Telescope (COAST) is a four aperture interferometric array being constructed at the University of Cambridge. It employs $0.4 \mathrm{~m}$ diameter telescopes and is designed to operate from $500 \mathrm{~nm}$ to $2.2 \mu \mathrm{m}$ with a maximum baseline of $100 \mathrm{~m}$. The building housing the path compensating optics and detector system is complete as are two of the four telescopes. It is expected that the first fringes will be obtained in 1990.

Other instruments which are under construction, or in an advanced design stage, include the Infrared-Optical Telescope Array (IOTA), a joint project involving the Smithsonian Astrophysical Observatory, Harvard, MIT, and the Universities of Wyoming and Massachusetts, the Astrometric Optical Interferometer (AOI) which is being constructed by the U. S. Naval Observatory, and the Big Optical Array (BOA) planned by the U.S. Naval Research Laboratory. The latter two instruments rely heavily on experience gained with the extremely successful Mark III Astrometric Interferometer on Mount Wilson, which was developed in a collaborative program between the Smithsonian Astrophysical Observatory, MIT, the U.S. Naval Research Laboratory and the U.S. Naval Observatory. 
Provision is being made in the design of the ESO VLT for the four $8 \mathrm{~m}$ telescopes to be operated in an interferometric mode, at least in the infrared. There are also plans to add an array of smaller telescopes with apertures of the order of $1 \mathrm{~m}$ diameter which may be used either as a separate imaging instrument or in conjunction with the larger telescopes. The final layout of the VLT and plans for the auxilliary array have not been finalised.

There are several proposed interferometers which have not yet been funded. These include the Center for High Angular Resolution Astronomy (CHARA) project of Georgia State University with baselines from $30 \mathrm{~m}$ to $300 \mathrm{~m}$ and seven apertures of $1 \mathrm{~m}$ diameter, the French VISIR project with baselines up to $300 \mathrm{~m}$ and at least three $1.5 \mathrm{~m}$ apertures, and the ambitious OVLA project of OCA which proposes twenty seven $1.5 \mathrm{~m}$ aperture telescopes in a ring configuration. All these proposed instruments are intended to operate at visual and infrared wavelengths.

In addition to the above plans several of the proposals for large aperture telescopes outlined in Section 2.1 include the possibility of interferometric use, in some cases through the addition of smaller auxilliary telescopes which can be moved relative to the large telescope to provide a range of baselines. An example is the NOAO proposal to include several mobile telescopes in the 1-2 $\mathrm{m}$ range as auxilliaries to an $8 \mathrm{~m}$ telescope to provide a very high angular resolution imaging capability.

\section{References}

Babcock, H.W., 1990. "Adaptive Optics Revisited". Science, 249, 253-257.

Bagnuolo, W.G., Furenlid, I.K., Gies, D.R., Barry, D.J., Russell, W.H., Dorsey, J.F., 1990. "The Multi-Telescope Telescope: A Cost-Effective Approach to Fiber-Fed Spectroscopy". Publ. Astron. Soc. Pacific, 102, 604-611.

Buscher, D.F., Haniff, C.A., Baldwin, J.E., Warner, P.J., 1990. "Detection of a bright feature on the surface of Betelgeuse". Mon. Not. R. Astron. Soc., 245, 7P-11P.

Forbes, F., Roddier, F., Poczulp, G., Sweeny, G., Dueck, R., 1989. "Segmented bimorph deformable mirror". J. Phys. E: Sci. Instrum., 22, 402-405.

Lacy, J.H., Achtermann, J.M., Bruce, D.E., Lester, D.F., Arens, J.F., Peck, M.C., Gaalema, S.D., 1989. "IRSHELL: A Mid-Infrared Cryogenic Echelle Spectrograph". Publ. Astron. Soc. Pacific, 101, 1166-1175.

Nather, R.E., Winget, D.E., Clemens, J.C., Hansen, C.J., Hine, B.P., 1990. "The Whole Earth telescope: A New Astronomical Instrument". Astrophys. J., (in press).

Papaliolios, C., Nisenson, P., Ebstein, S., 1985. "Speckle imaging with the PAPA detector". Applied Optics, 24, $287-$ 292.

Rousset, G., Fontanella, J.C., Kern, P., Gigan, P., Rigaut, Lena, P., Boyer, C., Jagourel, P., Gaffard, J.P., \& Merkle, F., 1990. "First diffraction-limited astronomical images with adaptive optics". Astron. Astrophys., 230, L29-32.

Roddier, F., 1988. "Curvature sensing and compensation: a new concept in adaptive optics". Applied Optics, 27,1223 1225 .

\section{Acknowledgements}

The unattributed sections of this report have been prepared with the help of information supplied by many people including: S.J. Adelman, J.C. Bhattacharyya, E.E. Becklin, P.M. Gray, J.-L. Heudier, C.M. Humphries, F. Merkle, and R.G. Tull.

John Davis

President of the Commission 